\section{Ocular coherence tomographic examination of postoperative foveal architecture after scleral buckling vs vitrectomy for macular off retinal detachment}

SK Gibran and PE Cleary
Department of

Ophthalmology,

Cork University Hospital,

Cork, Ireland

Correspondence: SK Gibran,

Tel: + 353214546400 ;

Fax: + 353214922656.

E-mail: syedgibran@

yahoo.com

Received: 12 January 2006 Accepted in revised form: 2 April 2006

Published online:

19 May 2006

No research funding and no proprietary interests

\begin{abstract}
Aims This pilot study uses Optical Coherence Tomography (OCT) imaging to compare the difference in foveal architecture after successful retinal detachment (RD) surgery by scleral buckling or pars plana vitrectomy (PPV).

Methods Prospective recruitment of patients with macular off RDs. Detachment surgery was undertaken by scleral buckling, external drainage, and air injection (group 1) or by PPV (group 2). Postoperatively patients had clinical examinations and OCT at 1, 3, 6, and 12 months. If abnormalities persisted, a further OCT was obtained at 18 months.

Results Retinal reattachment, including clinical macular reattachment, was achieved in all cases within $24 \mathrm{~h}$ postoperatively. In group $1(n=22)$, postoperative OCT showed persistent foveal detachment in $63 \%$ of cases $(n=14)$ at 1 and 3 months. At 6 and 12 months, $36 \%(n=8)$ and $9 \%(n=2)$ had a persistent foveal detachment, respectively, and at 18 months, foveal detachment eventually. In group $2(n=21)$, postoperative OCT showed an attached fovea in all cases; however, foveal thickening suggesting intraretinal oedema was present in all cases. The oedematous appearance of retina on OCT settled in 1-3 months. No foveal abnormality was seen at 6 and 12 months postoperatively.

Conclusions A high proportion of patients with successful retinal reattachment surgery by scleral buckling had foveal detachments
\end{abstract}

postoperatively. No cases who had PPV had foveal detachments; however, transient retinal oedema was evident in all cases. The aetiology of these changes is unknown and warrants further investigation, as there is the potential of a long-term effect on vision.

Eye (2007) 21, 1174-1178; doi:10.1038/sj.eye.6702429; published online 19 May 2006

\section{Introduction}

Persistent foveal detachment after successful primary retinal detachment (RD) surgery may partly contribute to delayed and incomplete postoperative visual recovery. ${ }^{1}$ Incidence of postoperative detachment was reported to be less by vitrectomy than by scleral buckling. ${ }^{2}$ Complete foveal reattachment after macular off RD occurs without delay after vitrectomy, whereas subfoveal fluid may persist subclinically for several months in patients operated with scleral buckling. ${ }^{3}$ We examined the postoperative foveal architecture by optical coherence tomography (OCT) in patients after successful scleral buckling and pars plana vitrectomy (PPV) procedures for macular involving primary rhegmatogenous $\mathrm{RD}$.

\section{Materials and methods}

In a prospective nonrandomised comparative study, we examined the postoperative foveal architecture by OCT in two groups of patients after successful scleral buckling (group 1) and PPV (group 2) procedures for primary RD. 
We prospectively recruited patients presenting to our service with clinically determined macular off rhegmatogenous RDs. Patients with known pre-existing ocular or macular pathology were excluded from the study. RDs with proliferative vitreoretinopathy were also excluded.

All patients underwent complete clinical examination with retinal drawings and OCT (six radial lines, OCT 2, Carl Zeiss Meditec AG, Germany). All OCTs were carried out by one experienced operator (SKG).

There was no difference in visual acuity (VA) preoperatively in both groups, as LogMar VA was 1.0.

In group 1, scleral buckling was performed using circumferential 277 silicone explant $(n=16)$ and half circle $5 \mathrm{~mm}$ sponge $(n=6)$. External drainage of subretinal fluid (SRF) was performed in 17 cases with air injection. Postoperatively, the patients were kept in lateral position for 5 days.

In group 2, all patients were treated with PPV, cryotherapy, complete fluid-air exchange, and 20\% sulphurhexafluoride (SF6) fill. Ten patients had 240 encirclement at the time of surgery. Perfluorocarbon was not used in any of these patients. Postoperatively, all patients were kept prone for $24 \mathrm{~h}$ and then in lateral position for 10 days.

All surgeries were carried out by both the authors (SKG \& PEC).

Patients were examined at 1 week, 1, 3, 6, and 12 months postoperatively with complete ophthalmic examination using indirect ophthalmoscopy and slit-lamp biomicroscopy. An OCT was performed at $1,3,6$, and 12 months. Topographic maps were produced by obtaining six consecutive cross-sectional scans at equally spaced angular orientations $\left(30^{\circ}\right)$ in a radial spoke pattern centred on the fovea. Therefore, retinal thickness measurements were performed with a total of 600 points along six intersecting lines with six measurements located in the central fovea (best possible fixation was obtained by patching the fellow eye of the patient, internal fixation was used, and it was confirmed with each patient that scan line intersected with the green target light). This pattern is, in theory, advantageous as the greatest concentration of measurements is within the central fovea where accurate measurements may be most important. The six scans were studied individually with the corresponding video image to verify the foveal cross-section. If abnormalities were detected at the final scan, a further OCT was obtained at the 18-month time point.

The Declaration of Helsinki was followed. Statistical analysis was by $t$-test utilising SPSS ${ }^{\circledR}$ for Windows 9.0.

\section{Results}

Forty-three consecutive patients were recruited into the study (31 men and 12 women; median age 65 (range 60-70) years).

Patient demographics are shown in Table 1.

In group 1 , seven patients $(31.8 \%)$ had a posterior vitreous detachment (PVD) documented preoperatively, whereas all patients in group 2 had preoperative PVDs.

In group 1, postoperative OCT showed persistent foveal detachment in $63 \%$ cases $(n=14)$ at 1 and 3 months, which was not visible clinically. Seven of these had a preoperative PVD, whereas seven had no evidence of preoperative PVD. At 6 months, eight cases (36\%) had a persistent foveal detachment (one of these had a preoperative PVD). At 12 months, foveal detachment

Table 1 Patient demographics

\begin{tabular}{lccc}
\hline & $\begin{array}{c}\text { Group 1 } \\
(\mathrm{n}=22)\end{array}$ & $\begin{array}{c}\text { Group 2 } \\
(\mathrm{n}=21)\end{array}$ & P-value \\
\hline Median age (range) & $64(60-69)$ & $67(63-70)$ & 0.103 \\
$\begin{array}{l}\text { Male: female } \\
\text { Phakic: pseudophakic }\end{array}$ & $18: 4$ & $14: 7$ & - \\
$\begin{array}{l}\text { Median duration of central } \\
\text { visual loss (range) }\end{array}$ & $6(3-7)$ & $5(3-7)$ & 0.329 \\
\hline
\end{tabular}

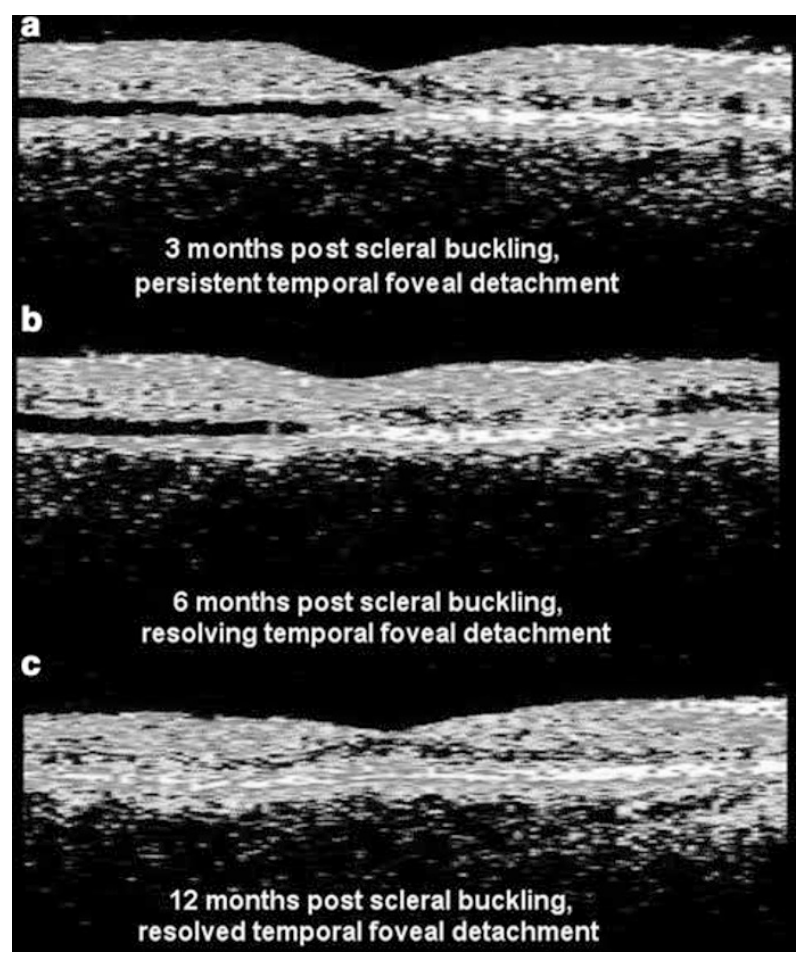

Figure 1 Typical optical coherence tomographic appearance of foveal detachment in cases treated with scleral buckling. 
persisted in two cases (9\%), which eventually resolved after 18 months (Figure 1). Neither of the latter cases had a pre-existing PVD.

In group 2, postoperative OCT showed an attached fovea in all cases; however, foveal thickening suggesting intraretinal oedema was present in all cases. The oedematous appearance of retina on OCT settled in

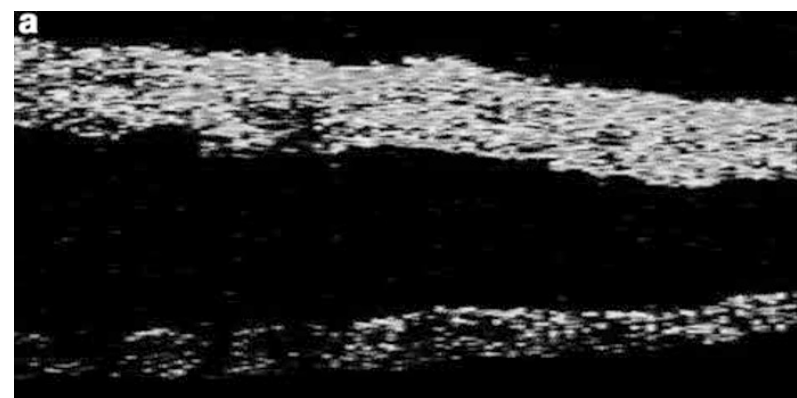

\section{Macular off RD with optic disc on right side}

\section{b}

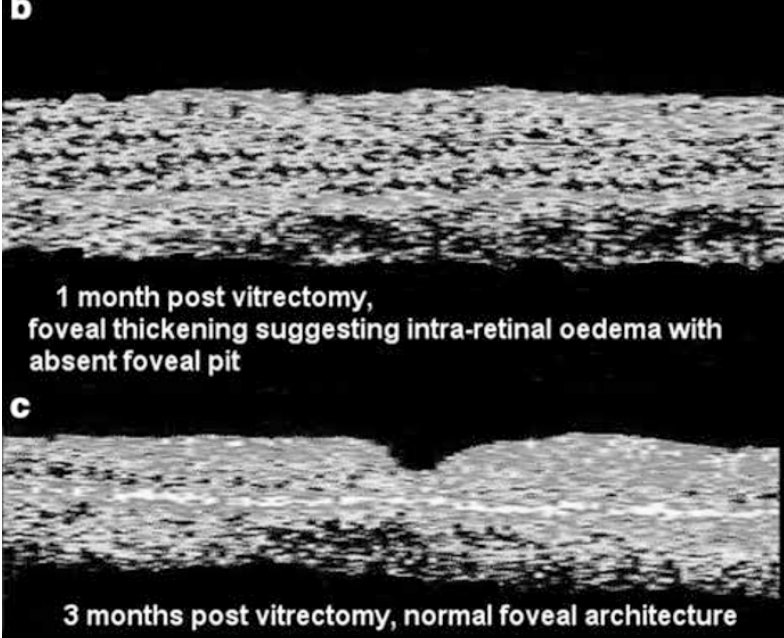

Figure 2 Typical optical coherence tomographic appearance of foveal oedema in cases treated with PPV.

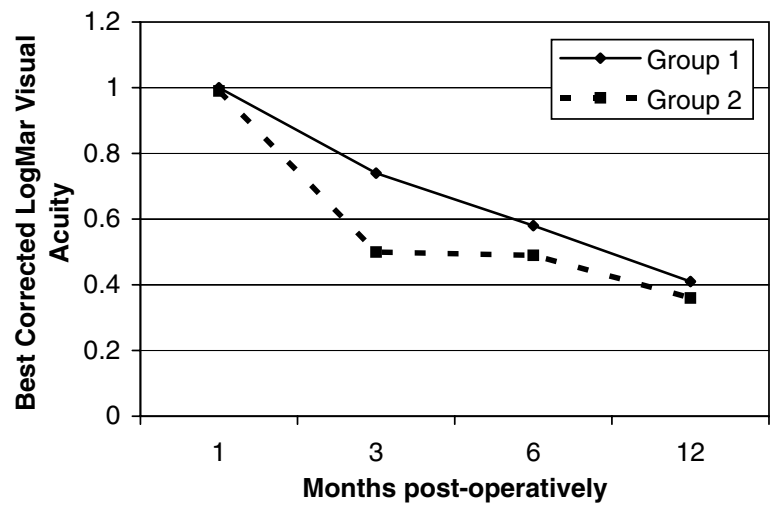

Figure 3 Chart showing postoperative best-corrected LogMar $\mathrm{VA}$ at each time point (months).
1-3 months. This finding disappeared at 6 and 12 months postoperatively (Figure 2).

Best-corrected LogMar pin-hole VAs for both groups at each postoperative time point are shown in Figure 3. There was no statistically significant difference in VA between the two groups at 1, 6, and 12 months postoperatively $(P=0.284,0.194$, and 0.317 , respectively). At 3 months, group 2 had a statistically significant better VA $(P<0.001)$. Resolution of the intraretinal oedema seen in group 2 did appear to be related to a significant step improvement in vision. A comparison of mean postoperative macular thickness (MT) and best-corrected LogMar pin-hole VA in group 2 patients is shown in Table 2.

In group 1, foveal reattachment was associated with a mean 0.27 LogMar unit improvement in pin-hole VA. Postoperative mean best-corrected LogMar pin-hole VA of group 1 patients with persistent foveal detachment is shown in Table 3.

\section{Discussion}

The aetiology of localised foveal detachment in patients after successful RD surgery is not clear. The presence of subfoveal fluid in macular-sparing $\mathrm{RD}^{4}$ and macular-involving $\mathrm{RD}^{1,5}$ has been noted previously. Spontaneous resolution of foveal detachment may take up to 12 months with corresponding postoperative improvement in VA.

Provided that all retinal breaks are identified and treated successfully, the source of subfoveal fluid should not be via the retinal break and thus its source remains speculative. Once adjacent retina has reattached fully, it would be expected that the retinal pigmentary epithelium (RPE) pump within the macular area would also facilitate rapid foveal reattachment. The subfoveal fluid may represent leakage from the RPE and/or choroidal blood vessels owing to alteration in the blood-retinal barrier; however, Wolfensberger and Gonvers ${ }^{6}$ showed with postoperative fluorescein angiography that there was no evidence of vascular leakage in such cases.

Another cause of fluid accumulation in the macula, which is more plausible, could be a localised loss of adherence of the RPE to Bruch's membrane (BM) owing to the surgical, trauma-induced, inflammation. In this case, the disturbance of the adherence between RPE and BM would theoretically permit access of fluid from the choroid to the subretinal space; however, in such an instance, the defect would be theoretically detectable on fluorescein angiography.

Scleral buckling has been documented to affect both subfoveal choroidal ${ }^{7}$ and retinal blood flow. ${ }^{8}$ These haemodynamic changes may alter the polarity of the RPE and lead to fluid leakage. Conversely, inflammation 
Table 2 Comparison of mean postoperative macular thickness and best-corrected LogMar pin-hole visual acuity in group 2 patients

\begin{tabular}{|c|c|c|c|c|c|}
\hline \multicolumn{2}{|l|}{1 month } & \multicolumn{2}{|c|}{3 months } & \multicolumn{2}{|c|}{6 months } \\
\hline$M T$ & $V A$ & $M T$ & $V A$ & $M T$ & $V A$ \\
\hline $\begin{array}{l}281.23 \\
P \text {-value }\end{array}$ & 0.89 & $\begin{array}{c}180.57 \\
0.0001 \text { vs } 1 \text { month }\end{array}$ & $\begin{array}{c}0.50 \\
0.0001 \text { vs } 1 \text { month }\end{array}$ & $\begin{array}{c}182.04 \\
0.1833 \text { vs } 3 \text { months }\end{array}$ & $\begin{array}{c}0.52 \\
0.6865 \text { vs } 3 \text { months }\end{array}$ \\
\hline
\end{tabular}

MT = mean macular thickness in $\mu \mathrm{m} ; \mathrm{VA}=$ best-corrected LogMar pin-hole visual acuity.

Table 3 Postoperative mean best-corrected LogMar pin-hole visual acuity of group 1 patients with persistent foveal detachment

\begin{tabular}{|c|c|c|c|c|c|c|c|c|c|c|}
\hline \multirow[t]{2}{*}{ Pt. } & \multicolumn{2}{|c|}{1 month } & \multicolumn{2}{|c|}{3 months } & \multicolumn{2}{|c|}{6 months } & \multicolumn{2}{|c|}{12 months } & \multicolumn{2}{|c|}{18 months } \\
\hline & $V A$ & $S R F$ & $V A$ & $S R F$ & $V A$ & $S R F$ & $V A$ & $S R F$ & $V A$ & $S R F$ \\
\hline 2 & 1 & + & 0.8 & + & 0.5 & - & 0.3 & - & 0.3 & - \\
\hline 4 & 1 & + & 1 & + & 0.8 & + & 0.5 & - & 0.5 & - \\
\hline 5 & 1 & + & 1 & + & 0.8 & + & 0.6 & + & 0.6 & - \\
\hline 6 & 1 & + & 1 & + & 0.6 & - & 0.5 & - & 0.5 & - \\
\hline 8 & 1 & + & 1 & + & 0.8 & - & 0.3 & - & 0.3 & - \\
\hline 10 & 1 & + & 0.8 & + & 0.5 & - & 0.5 & - & 0.5 & - \\
\hline 11 & 1 & + & 0.8 & + & 0.5 & - & 0.3 & - & 0.3 & - \\
\hline 12 & 1.00 & + & 1 & + & 1 & + & 0.8 & - & 0.8 & - \\
\hline 14 & 1 & + & 1 & + & 1 & + & 0.8 & + & 0.5 & - \\
\hline 16 & 1 & + & 0.8 & + & 0.6 & + & 0.5 & - & 0.5 & - \\
\hline 17 & 1 & + & 0.8 & + & 0.5 & - & 0.3 & - & 0.3 & - \\
\hline 18 & 0.8 & + & 0.8 & + & 0.8 & + & 0.5 & - & 0.5 & - \\
\hline 20 & 1 & + & 0.8 & + & 0.6 & + & 0.5 & - & 0.5 & - \\
\hline 22 & 1 & + & 0.8 & + & 0.6 & + & 0.5 & - & 0.5 & - \\
\hline
\end{tabular}

$\mathrm{VA}=$ best-corrected LogMar pin-hole visual acuity; SRF = subfoveal fluid; + = SRF present; =SRF absent.

induced by scleral buckling itself may be the source of the subfoveal fluid. ${ }^{5,9}$

It is also possible that the remaining vitreous body may play a role in maintaining serous elevation of fovea. Air injected into the vitreous of an eye without a PVD could theoretically lead to vitreomacular traction and contribute to persistence of foveal detachment. Half of the cases with persistent foveal detachment 3 months postoperatively had a preoperative PVD. By the 6-month time point, only one of the patients with a preoperative PVD had a foveal detachment. Both the cases with persistent foveal detachment 1 year postoperatively had attached vitreous.

Interestingly in our study, there were no cases of foveal detachment in patients who were treated with PPV and gas fill. These patients showed intraretinal oedema postoperatively on OCT, which tended to resolve by 1-3 months; however, none had true foveal detachment on any of their OCT scans. All of these patients had complete resolution of the retinal oedema after 3 months postoperatively. This is in stark contrast to the $63 \%$ of subjects in the scleral buckling group who had foveal detachment in the first 3 months postoperatively. This feature lends credence to the theory that it may be the remaining vitreous that is responsible for the postoperative foveal detachment, particularly, as all of the PPV patients had a pre-existing PVD. It seems reasonable to hypothesise that maximum removal of vitreous with complete fluid air exchange and postoperative prone posturing for $24 \mathrm{~h}$ may help to evacuate the subfoveal fluid.

The presence of transient intraretinal oedema in the PPV group is probably owing to the continuous irrigation during the procedure and short-term surgical-induced inflammation. A transient increase in MT, parallel with decrease in VA, also occurs after uneventful PPV for troublesome vitreous floaters (unpublished data). However, this does not appear to preclude satisfactory improvement of VA. Resolution of the intraretinal oedema seen in group 2 did appear to be related to a significant step improvement in vision, whereas, in contrast, in group 1, foveal reattachment was associated with a mean 0.27 LogMar unit improvement in pin-hole VA.

At 1 month postoperatively, there was no statistically significant difference in VA of two groups because, in group 1, approximately $2 / 3$ of patients had subclinical foveal detachment, and in group 2, all patients showed foveal thickening on OCT (Table 2).

At 3 months postoperatively, the PPV group had a statistically significant better VA. This difference may be hypothesised to be related to the presence of persistent 
foveal detachment in almost two-thirds of the scleral buckling group and resolution of foveal thickening in group 2 patients.

An inverse relationship between postoperative visual recovery and persistent foveal detachment has been noted previously. ${ }^{4,5}$ It is logical to presume that duration of postoperative foveal detachment may play a role in patients whose vision takes longer to improve. The chronicity of foveal detachment may predispose to photoreceptor damage and explain cases of incomplete postoperative visual recovery after successful RD repair following scleral buckling. Despite apparent recovery of central acuity with time, it is clear in modern ophthalmology that BCVA is not a definitive marker of success. Other visual parameters, such as contrast sensitivity for example, may be adversely affected by prolonged foveal detachment despite apparent restoration of premorbid acuity. The correlation of the postoperative foveal detachment to other visual parameters may be an avenue for further investigation.

As clinical examination alone may fail to reveal the presence of foveal detachment, the authors suggest that OCT is the tool of choice in investigating the delayed postoperative visual recovery in such cases; however, the role of potential interventions to speed visual recovery remains speculative and will only become apparent with further study.

The most crucial question is how to prevent the postoperative foveal detachment in the externally repaired cases, as it is unlikely to be a completely innocuous phenomenon. We acknowledge that our pilot study does not answer the question of aetiology; however, our data do suggest that the residual vitreous may be a causative factor presumably owing to tractional forces and support previously reported literature that in macular off RDs, foveal attachment seems to be faster by PPV approach as compared to scleral buckling procedures. ${ }^{6}$ Further research is required to assess whether the presence of pathological subfoveal fluid does indeed predispose to an, albeit small, detrimental effect on final outcome with regard to VA or visual performance as a whole. Certainly, further work should be undertaken to determine whether the onset and duration of this foveal detachment may be modified with measures such as postoperative prone posturing or the use of steroids to prevent any inflammatory component to the pathogenesis. To establish these facts further, a large prospective randomised controlled trial is necessary.

\section{References}

1 Hagimura N, Iida T, Suto K, Kishi SL. Persistent foveal retinal detachment after successful rhegmatogenous retinal detachment surgery. Am J Ophthalmol 2002; 133: 516-520.

2 Kambara C, Inoda S, Shimiza Y. Optical coherence tomographic features after surgery for rhegmatogenous retinal detachment with macular involvement. Jpn J Clin Ophthalmol 2000; 54: 327-330.

3 Thomas J, Wolfensberger TJ. Foveal re-attachment after macula-off retinal detachment occurs faster after vitrectomy than after buckle surgery. Ophthalmology 2004; 111: 1340-1343.

4 Theodossiadis PG, Georgalas IG, Emfietzoglou J, Yriaki TE, Pantelia E, Gogas PS. Optical coherence tomography findings in the macula after treatment of rhegmatogenous retinal detachments with spared macula preoperatively. Retina 2003; 23: 69-75.

5 Baba T, Hirose A, Moriyama M, Mochizuki M. Tomographic image and visual recovery of acute macula-off rhegmatogenous retinal detachment. Graefes Arch Clin Exp Ophthalmol 2004; 242: 576-581.

6 Wolfensberger TJ, Gonvers M. Optical coherence tomography in the elevation of incomplete visual recovery after macula-off retinal detachments. Graefes Arch Clin Exp Ophthalmol 2002; 240: 85-89.

7 Regillo CD, Sergott RC, Brown GC. Successful scleral buckling procedures decrease central retinal artery blood flow velocity. Ophthalmology 1993; 100: 1044-1049.

8 D'Hermies F, Korobelnik JF, Chauvaud D, Pouliquen Y, Parel JM, Renard G. Scleral and episcleral histological changes related to encircling explants in 20 eyes. Acta Ophthalmol Scand 1999; 77: 279-285.

9 Birgul T, Vidic B, El-Shabrawi Y. Intrusion of an encircling buckle after retinal detachment surgery. Am J Ophthalmol 2003; 136: 942-944. 